\title{
Children's Understanding of Depression
}

By Niki Georgakakou-Koutsonikou, Emily Taylor \& Jo Williams

The authors are based at the Centre for Applied Developmental Psychology, Clinical and Health Psychology, School of Health in Social Sciences, University of Edinburgh.

This article is a summary of the paper published in CAMH - Georgakakou-Koutsonikou N, Taylor EP, Williams JM. (2019). Children's concepts of childhood and adolescent depression. Child and Adolescent Mental Health, 24(1), 19-28. doi: 10.1111/camh.12266

Depression is a mental illness that affects children and especially adolescents, however, little is known about how children and adolescents understand depression. Gaining an understanding of how children perceive illness can facilitate effective communication with health professionals and children's active involvement in decision-making about their health. Research on children's understanding of mental illness can improve our understanding of stigma and peer exclusion and inform the development of tailored psychoeducational interventions. To date, there is considerably less focus on children's understanding of mental health in comparison to physical health, and less research on children's understanding than on adolescent groups.

The aim of this study was to explore how children understand depression as it affects children and adolescents. We also wanted to examine whether age, gender and experience of depression, or contact with people with depression, is related to children's understanding.

To address these questions, we conducted individual interviews with 105 children, 38 children aged 8-9 years, and 67 children aged $11-12$ years. To introduce the topic, we created three short stories describing fictional child and adolescent characters with depression, as well as a control story of a character without depressive symptoms. These were read to each child, who was then asked a standardised series of questions. The foci of the questions were based on the model of illness representations, which suggests that there are five dimensions to a cognitive representation of illness:

1) Identity (its symptoms and label),

2) Causes,

3) Consequences (impact of the illness),

4) Curability

5) Timeline to recovery.

To analyse the interview data, we performed content analysis for each of the five categories of the illness representation model, by finding common themes in children's responses. This analysis was then followed by quantitative content analysis techniques and statistical testing.

The majority of children identified that the characters in the stories had a difficulty of a psychological nature, in comparison to the control story. Approximately half of the children considered this difficulty to be a mental health problem, and less than one-fifth were able to label this difficulty as depression. Children considered various causes of depression, which were primarily psychosocial. 
The causes of perceived depression were grouped in six categories:

1) Peer relationships (mentioned by $45 \%$ of children) include bullying, disputes with peers and loneliness.

2) Family factors (37\%) involve parental divorce, disputes and being unloved or neglected by parents.

3) Individual factors (32\%) reflect the child's internal state, involving factors such as negative thinking, extensive worrying and low self-esteem.

4) Physiological factors (16\%), included fatigue, sleep difficulties, poor appetite, physical illness and puberty.

5) Factors related to school (17\%), such as exam stress or transitions to secondary school.

6) Loss was considered a cause by one in every ten children (10\%).

Most children considered depression to be curable $(87 \%)$ or possibly curable (11\%), within a short period of time. Specifically, the percentages of responses in each duration category were: up to one day (3\%), a few days ( $12 \%)$, a couple of weeks (18\%), one to two months $(34 \%)$, more than two months to less than a year (21\%), a year (6\%), and more than a year (5\%).

If depression is left untreated, children anticipated primarily negative outcomes. Perceived negative consequences of depression involved: deterioration of emotional state (30\% of participants), interpersonal difficulties in friendships and interactions with peers, including bullying ( $15 \%)$, decline of school performance or school refusal (14\%), behavioural consequences (11\%) such as disengagement from activities, changes in sleep and appetite, potential risk ( $9 \%)$ including self-harm and suicidality ( $9 \%$ ) and cognitive consequences, including negative thinking or self-beliefs (6\%). Finally, long-term consequences ( $9 \%$ included negative career prospects, financial difficulties and general underachievement.

Older children's concepts were more sophisticated than younger children's. Eleven to twelve-yearolds were more able to recognise depression, categorising depression as a mental health problem and differentiating between clinical depression and control conditions. Older children suggested a larger number of causes for each character and anticipated more negative consequences of depression and a longer period to recovery. Gender and experience (either personal or through contact) were not systematically associated with how children conceptualise depression.

Children from the age of 8-9 years are able to identify emotional difficulties displayed by hypothetical characters and demonstrate detailed concepts of depression that align more closely with clinical conceptualisations with age. Some differences between children's and professionals' conceptualisations of

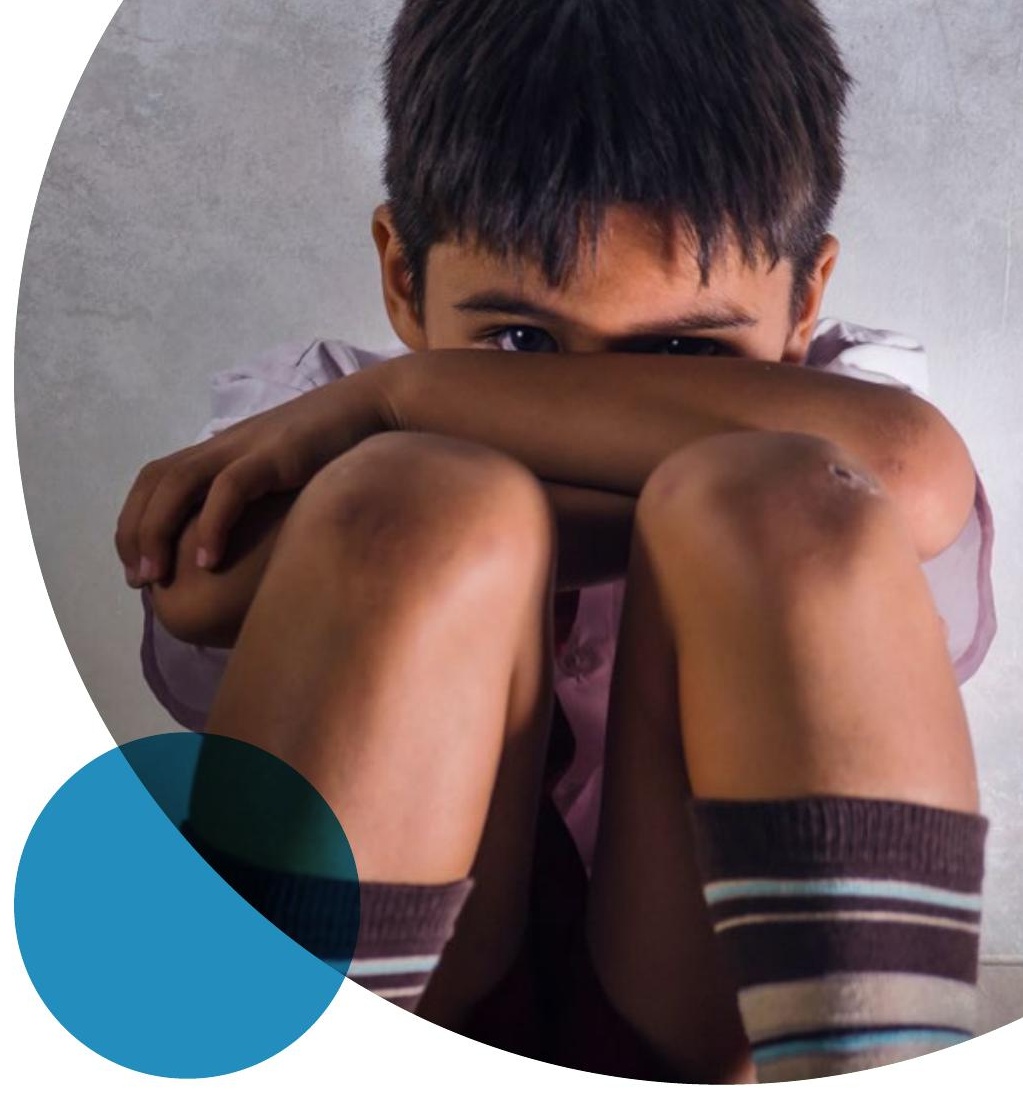

depression are evident, including the categorisation of depression as a mental illness, and the perceived timeline to recovery.

In clinical practice, it is important to take into consideration children's beliefs and to target misconceptions. Clinicians working with children with depressive symptoms should consider exploring children's understanding and treatment expectations as part of the treatment process. Surprisingly, contact or experience was not related to children's understanding of depression. Clinicians should therefore be aware that children in this age group who have contact with a person with depression (e.g. parent or sibling) do not necessarily have more knowledge about depression than their peers with no contact.

In this study, it is evident that children might use the term depression to refer to the emotional state of feeling low, rather than a psychological disorder. In clinical practice and in mental health education, defining terms used for mental health problems that are also commonly used in everyday language to describe emotional states (e.g. depression, anxiety) would facilitate shared understanding.

This is the first study to explore in detail how children think about depression. A limitation of this study is the fictional nature of the characters used; it is unknown whether children's concepts would equal personal beliefs if experiencing depression. Further, differences between the three stories used are difficult to interpret due to the study design. Cultural and ethnic differences were not examined in this study. Finally, this study examined children's understanding of depression affecting children or adolescents; children's views of depression affecting adults might differ. 
Key implications for practice:

- The results suggest that CAMHS practitioners should consider children's views of depression in their communications about diagnosis, prognosis and treatment of depression

- There is also a need for preventative school-based interventions to enhance children's understanding of depression and address the some misconceptions that children hold about mental illness

- Further scientific research is required on children's understandings of childhood mental illnesses and psychological difficulties such as depression, anxiety, conduct disorders and learning difficulties.

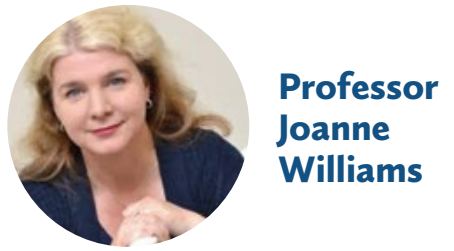

I am an applied developmental psychologist with research interests in child and adolescent health and mental health, the development of health concepts, developmental disabilities and children's interactions with animals.

Since 2012 I have been Senior Lecturer/Professor of Applied Developmental Psychology in Clinical and Health Psychology. I coordinate Children, Adolescents and Animals Research (caar), and I am Deputy Director of the Centre for Applied Developmental Psychology.

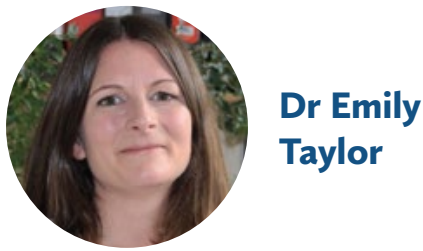

I joined the School of Health in Social Science in September 2010, where I am Programme Director for the postgraduate programmes in Children and Young People's Mental Health and Psychological Practice and the PGCert cyCBT. I continue to work in NHS Lothian in a CAMHS outpatient team, providing CBT and IPT. (bio via www.ed.ac.uk)

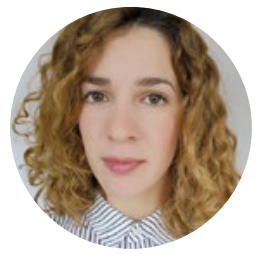

\section{Dr Niki \\ Georgakakou- Koutsonikou}

I began my PhD in Clinical Psychology at the University of Edinburgh in 2013 examining children and adolescents' conceptualisations of depression.

My research interests include children and young people's understanding of mental health and illness, mental health stigma and help-seeking. 